\title{
Cahiers d'études africaines
}

$238 \mid 2020$

Varia

PETERSON Derek R., HUNTER Emma \& NEWELL

Stephanie (eds.). - African Print Cultures. Newspapers and Their Publics in the Twentieth Century

Florence Brisset-Foucault

\section{(2) OpenEdition}

Édition électronique

URL : https://journals.openedition.org/etudesafricaines/30637

DOI : 10.4000/etudesafricaines.30637

ISSN : $1777-5353$

Éditeur

Éditions de l'EHESS

Édition imprimée

Date de publication : 15 juin 2020

Pagination : 455-459

ISBN : 978-2-7132-2828-5

ISSN : 0008-0055

Référence électronique

Florence Brisset-Foucault, « Peterson Derek R., hunter Emma \& Newell Stephanie (eds.). - African Print Cultures. Newspapers and Their Publics in the Twentieth Century ", Cahiers d'études africaines [En ligne], 238 | 2020, mis en ligne le 15 juin 2020, consulté le 04 janvier 2023. URL : http:// journals.openedition.org/etudesafricaines/30637 ; DOI : https://doi.org/10.4000/etudesafricaines. 30637

Ce document a été généré automatiquement le 4 janvier 2023

Tous droits réservés 


\title{
PETERSON Derek R., HUNTER Emma \& NEWELL Stephanie (eds.). - African Print Cultures. Newspapers and Their Publics in the Twentieth Century
}

\author{
Florence Brisset-Foucault
}

\section{RÉFÉRENCE}

Peterson Derek R., hUNTER Emma \& Newell Stephanie (eds.). - African Print Cultures. Newspapers and Their Publics in the Twentieth Century. Ann Arbor, The University of Michigan Press, 2016, 460 p., index, ill.

1 Ce beau livre collectif, au-delà du plaisir qu'il procure via la découverte d'une quinzaine d'études de cas minutieusement documentées et couvrant une très large période (de la fin $d u X X^{e}$ au début du $X X^{e}$ siècle), représente une avancée épistémologique et méthodologique bienvenue au regard des travaux existants sur les médias en Afrique. Il est d'abord un antidote contre une approche très courante de la presse sur le continent comme «baromètre de la démocratie », qui évalue les médias selon des modèles et des valeurs dont l'historicité n'est que très peu questionnée, et qui a tendance de ce fait à les décrire largement sur le registre de l'échec ou du manque. Ces travaux se révèlent, par conséquent, incapables de rendre compte de l'ambivalence de ces médias et de leurs effets, ou de rendre justice au potentiel heuristique de l'étude de pratiques et de conventions de travail journalistiques qui peuvent paraitre hétérodoxes au regard de certains modèles, mais qui caractérisent de facto la production médiatique ${ }^{1}$.

2 Ainsi, par exemple, de la réimpression de coupures issues d'autres journaux, analysée par Leslie James (pp. 49-74) et courante dans la presse du Nigeria des années 1930 et 1940. Celle-ci aurait pu être disqualifiée d'emblée comme typique de journaux pauvres, inexpérimentés, qui n'étaient pas «réellement» engagés dans le journalisme. Ces 
pratiques étaient, cela ne fait aucun doute, le produit de la nécessité. Cependant, elles offraient aussi des opportunités «nichées dans le genre lui-même" (p.66) et demandent, de ce fait, à être interrogées sur ce qu'elles révèlent non seulement du fonctionnement et de l'économie de la presse, mais aussi des "affinités déterritorialisées " (ibid.) qu'elles reflètent et nourrissent. De même, l'attention aux genres apparemment mineurs de la presse s'avère extrêmement enrichissant : ainsi du superbe chapitre de Stephanie Newell (pp.389-424) sur les nécrologies qui clôt l'ouvrage (mais on aurait pu aussi inclure d'autres genres négligés tels que les recettes de cuisine et autres rubriques domestiques, ou la publicité). L'ouvrage contribue également à remédier à la marginalisation historiographique de la presse d'État, souvent négligée. À rebours de l'idée qu'il s'agirait de simples reflets de l'État colonial, sans aucune autonomie et donc, par définition, inintéressants à étudier, c'est précisément cette "agency in tight corners $»^{2}$ sur laquelle il est nécessaire de se pencher (capacité d'action réduite qui peut passer, par exemple, par la fiction, ou des genres qui semblent a priori apolitiques comme la poésie ou la nécrologie déjà citée). Mais ce sont également les conditions du consentement qui devraient nous interpeler, ainsi que le mélange parfois non intentionnel entre résistance et adhésion qui est fascinant à reconstituer. Cela, Uta Reuster-Jahn parvient admirablement à le faire dans le chapitre 8 sur la presse de divertissement en Tanzanie dont les créateurs, en célébrant des modes de vie du quotidien urbain, se positionnent un peu malgré eux puisqu'ils cherchent plutôt à éviter tout conflit avec l'État, en contrepoint de la promotion du socialisme rural de l'Ujamaa.

Dans la veine des travaux de Karin Barber dont les auteurs sont très proches de par leur démarche, le livre est une occasion d'explorer l'invention, la stabilisation et parfois même le retournement (dans le cas, là encore, des nécrologies) de certains genres. Les auteurs sont vigilants à les dénaturaliser et à reconstituer leur genèse, à la croisée de multiples influences sociales endogènes et de réseaux transnationaux. Les genres ne sont pas fermés. Le livre encourage à ne pas considérer ces objets en isolement, mais plutôt, comme l'avait déjà fait Marie-Soleil Frère dans son étude comparée de la presse au Niger et au Bénin ${ }^{3}$, comme des points de rencontre de cultures diverses de l'écrit et de l'imprimé, mais aussi de la performance, de l'art oratoire, sous l'influence des institutions religieuses ou scolaires. Cette continuité et cette porosité entre les genres parcourent toutes les études de cas, entre journal, roman, théâtre, pamphlet, cinéma. Ainsi des romans photos, ou plutôt du "théâtre photo", étudié par Olubukola Gbadegesin, qui cherche, à travers différentes techniques, à reproduire la dramaturgie et le mouvement caractéristiques du théâtre yoruba à travers l'image et le texte (via l'usage du flou notamment) (chapitre 9, pp. 251-280).

4 Par ailleurs, l'ouvrage engage à l'exploration de ce que permettent ou entrainent les journaux en termes de sociabilités, que ce soit à travers l'analyse des kamukunji d'Eldoret, ces parlements de lecteurs étudiés par Duncan Omanga dans le Kenya contemporain (déjà étudiés dans un article paru dans Politique africaine ${ }^{4}$, ou à travers celle des «sociabilités de l'imprimé » étudiées par Rebecca Jones dans le Nigeria des années 1930. Ces sociabilités sont mises en scène à travers la publication de récits de voyages et la description de successions de rencontres entre des animateurs de journaux et leurs lecteurs à travers le pays.

5 De manière plus classique, l'ouvrage vient utilement compléter l'appréhension historienne de la presse comme source de compréhension de la pensée politique sur le 
continent. Cette approche a déjà donné lieu à des travaux fructueux sur les pensées variées de la légitimité politique, de l'émancipation, de l'honneur, en tant qu'homme, femme, sujet de Dieu, travailleur, citoyen du monde, d'une nation ou en tant que patriote ethnique ${ }^{5}$. Dans la lignée du renouveau des débats sur les nationalismes africains, le livre se positionne fortement contre l'idée d'une relation univoque entre l'invention de l'imprimé et la construction nationale : de par le processus même de leur fabrique, au carrefour de réseaux transnationaux et transcontinentaux, ces journaux sont nourriciers d'un certain cosmopolitisme, y compris bien sûr dans le cas des publications en langues vernaculaires. Ainsi, l'imprimé ne promeut pas que de la nation, mais parfois des communautés politiques plus restreintes ou qui la débordent. Il peut aussi promouvoir des formes de nations alternatives, voire controversées, en tout cas largement débattues. Les «publics » (on pourrait discuter de l'usage du terme) ou les communautés politiques convoqués par ces publications sont instables, controversés et multiples, y compris au sein du même journal. Ainsi, plusieurs contributions, dont celle par exemple de David Pratten (chapitre 3), font le pari de donner à voir l'hétérogénéité et même le caractère éclectique des espaces conceptuels, langagiers et de positionnements politiques créés par ces publications plutôt que de dégager, de prime abord, des convergences ou une cohérence. Par ailleurs, les journaux encouragent bien souvent la reconnaissance mutuelle d'une "communauté de lecteurs » sur la base de critères de distinction sociale de classe. Frontières de classe dont la rigidité réaffirmée par certains de ces journaux est soulignée via l'exemple de ces membres de l'élite saro décrits par Karin Barber (chapitre 6) qui, au nom de la préservation de leur potentiel d'interpellation et de dialogue avec les autorités coloniales, se prémunissent d'utiliser un anglais plus populaire et plus accessible qui leur aurait permis la convocation d'une audience plus large. Au sein même de l'espace public nigérian particulièrement riche qui constitue le cœur de l'ouvrage, la présence du chapitre rédigé par Oluwatoyin Babatunde Oduntan (chapitre 11) est particulièrement bienvenue : portant sur une publication « de province », " aux marges de la société coloniale ", il permet de compléter et de mettre en perspective la pièce lagosienne au sein de la vaste mosaïque que constitue cet espace public hétérogène, dont les différentes composantes sont cependant en constante interaction. Il permet de sortir d'une histoire de l'imprimé cantonnée aux capitales ou aux plus grands pôles urbains, d'y intégrer des espaces plus larges, voire ruraux, et de donner une vision plus complète de l'histoire intellectuelle de ces sociétés.

6 Mais encore une fois, les journaux ne sont pas simplement des sources pour accéder à des contenus, et le livre invite à explorer et à reconstituer l'épaisseur de ce processus complexe de la fabrication des cultures de l'imprimé - ce qui vient encore enrichir la compréhension des pensées politiques du continent. Les pratiques de " copier-coller ", de re-publication, de réutilisation, déjà évoquées, mais aussi les effets formels et visuels de mise en page, de typographie, sont au cœur de plusieurs chapitres (notamment les chapitres 2, 9 et 14). Ainsi, des articles pris isolément n'ont que peu de signification ou de pouvoir d'interpellation, mais leur association leur donne une dimension subversive, sans même besoin de commentaire textuel d'un journaliste. La juxtaposition offre de nouvelles possibilités critiques dans des contextes de contrainte. Le livre illustre à merveille toute l'importance d'interroger cette matérialité, y compris la plus triviale. Ainsi, le livre constitue une mise en garde contre toute forme de déterminisme politique, et rappelle la nécessité de prendre en compte ce qui ne se réduit pas au politique, à l'action de l'État, dans la fabrique de l'imprimé, y compris dans un contexte 
d'oppression coloniale et autoritaire. Les différents auteurs prennent au sérieux ce qui relève, aussi, de la routine du travail, des contraintes et opportunités matérielles, d'une ambition artistique, professionnelle, sociale. Ainsi, avec ces précautions en tête mais dans une perspective qui s'intéresse plus directement à l'exercice du pouvoir, on aurait $\mathrm{pu}$ vouloir explorer davantage les tensions, les négociations autour des langages médiatiques et les tâtonnements avec les frontières de la décence ou de l'insolence, non seulement avec les autorités de l'État (et tous ses agents parfois antagonistes), mais aussi avec d'autres pôles de pouvoir et de conformisme (partis politiques, églises, entreprises qui interviennent via les marchés publicitaires). Sans pour autant étudier ces négociations dans le cadre de pensée normatif et souvent étriqué de la " démocratisation » ni interpréter ces pratiques de manière univoque comme autant de signes de «résistance». Un angle d'analyse plus politique, qu'on pourrait rapprocher d'une démarche telle que celle de Robert Darnton sur la censure, aurait fourni un axe de travail complémentaire et sans doute particulièrement fructueux ${ }^{6}$.

L'ouvrage aurait pu aussi aller plus loin dans l'exploration de l'épaisseur de la fabrique de l'imprimé. On sait ce que ces journaux doivent à l'engagement individuel d'un intellectuel ou d'un notable, cependant on s'interroge sur ce qu'Howard Becker appelle le "personnel de renfort", ces "petites mains » invisibles qui interviennent dans le processus de fabrication et dont l'action est souvent révélatrice de dimensions structurelles de la fabrique de ces cultures de l'imprimé et des espaces publics (les acteurs des "photo plays", les photographes, les techniciens divers, les lecteurs qui écrivent, les vendeurs, contre qui d'ailleurs la répression est souvent tournée) ${ }^{7}$. Par ailleurs, cet ouvrage concentré sur l'Afrique anglophone et qui fait la part belle au Nigeria incite à penser de manière comparée la situation dans les autres Afriques. En Afrique francophone notamment, de manière générale, même s'il faudrait évidemment nuancer (avec l'exemple du Bénin notamment), les cultures de l'imprimé se sont développées plus tard, et sans doute de manière plus articulée avec l'État colonial, même si bien souvent sur le modèle de la tension. Ce n'est pas le moindre apport de cet ouvrage que d'encourager, y compris par ces manques, à ouvrir tout un programme de recherche qui étendrait l'amplitude empirique à la fois à des acteurs négligés (le « personnel de renfort »), à d'autres espaces géographiques, et à l'étude de l'exercice du pouvoir.

\section{NOTES}

1. On pense, par exemple, à ce que l'on a observé au Buganda dans les années 1950, c'est-à-dire l'usage des journaux comme outils de surveillance voire de chantage, notamment via la dénonciation, avec force détails sur l'identité (adresse, le numéro de plaque de voiture) des briseurs du boycott promu contre les Britanniques et les Asiatiques par les partis royalistes.

2. J. LoNSDAlE, "Agency in Tight Corners : Narrative and Initiative in African History ", Journal of African Cultural Studies, 13 (1), 2000, pp. 5-16. 
3. M.-S. F RÈRE, Presse et démocratie en Afrique francophone: les mots et les maux de la transition au Bénin et au Niger, Paris, Karthala, 2000.

4. J. RASMUSSEN \& D. OMANGA, «Les parlements du peuple au Kenya: débat public et participation politique à Eldoret et Nairobi », Politique africaine, 127 (3), 2012, pp. 71-90.

5. On pense, par exemple, aux travaux de J. GLASSMAN, War of Words, War of Stones : Racial Thought and Violence in Colonial Zanzibar, Bloomington, Indiana University Press, 2011.

6. R. DARNTON, Censors at Work: How States Shaped Literature, New York, Norton, 2014.

7. H. BECKER, Les mondes de l'art, Paris, Flammarion, 1988. 